\title{
Tip II Genelleştirilmiş Çan Şekilli Bulanık Sayısının Tip II Parametrik Yamuk Bulanık Sayı Yakınsanması
}

\author{
Sinem PEKER ${ }^{* 1}$, Efendi N. NASİBOV ${ }^{2}$ \\ 1Yaşar Üniversitesi, Fen-Edebiyat Fakültesi, Matematik Bölümü, 35100, İzmir, Türkiye \\ (ORCID: https://orcid.org/0000-0003-4700-7595) \\ ${ }^{2}$ Dokuz Eylül Üniversitesi, Fen Fakültesi, Bilgisayar Bilimleri Bölümü, 35390, İzmir, Türkiye \\ (ORCID: https://orcid.org/0000-0002-1889-6410)
}

(Alınıș / Received: 03.10.2018, Kabul / Accepted: 25.12.2018, Online Yayınlanma / Published Online: 24.04.2019)

\section{Anahtar Kelimeler}

Tip II bulanık sayl,

Yakınsama,

Parametrik yamuk bulanık sayl

\begin{abstract}
Özet: Belirsizliğin olduğu durumlarda bulanık sayının 0 ve 1'den farklı olarak diğer üyelik seviyelerine olanak tanıması çeşitli uygulamalarda bulanık sayının kullanılmasına yol açmıştır. Tip 1 bulanık sayılarda her bir x değerine bir üyelik seviyesi ataması yapılmaktadır. Ancak her bir x değerinin üyelik seviyesine sadece bir üyelik değeri atanmasının zor olduğu durumlar olabilir. Bu gibi durumlarda her bir x değerine bulanık bir sayı șeklinde üyelik derecesi atanmasına olanak tanıyan Tip II bulanık sayıları kullanılabilir. Bulanık sayı ișlemlerinde, karmașı tipte bulanık sayılar kullanıldığında bazen zorluklar yașanabilir ve daha basit yapıdaki bulanık sayılarla çalıșılmak istenebilir. Bu çalışmada Tip II genelleștirilmiş çan ve daha basit yapıdaki Tip II parametrik yamuk bulanık sayıları ele alınmış ve Tip II genelleştirilmiş çan bulanık sayısının Tip II parametrik yamuk yakınsaması oluşturulmuştur.
\end{abstract}

\section{Type II Parametric Trapezoidal Approximation of Type II Generalized Bell-Shaped Fuzzy Number}

\section{Keywords}

Type II fuzzy number, Approximation, Parametric trapezoidal fuzzy number

\begin{abstract}
The fact that fuzzy numbers allows other levels of membership except from 0 and 1 lead to the use of fuzzy numbers in various applications in cases where there is uncertainty. In type 1 fuzzy numbers, a crisp membership level is assigned to each $\mathrm{x}$ value. However, there may be cases where it is difficult to assign only one membership value to the membership level of each $x$ value. In such cases, Type II fuzzy numbers can be used, which allow a fuzzy number membership value to be assigned to each $\mathrm{x}$ value. When using complex type fuzzy numbers in fuzzy number operations, sometimes there may be difficulties and it may be desirable to work with fuzzy numbers in a simpler structure. In this study, Type II generalized bell-shaped, Type II parametrical trapezoidal fuzzy numbers in simpler form are considered, and Type II parametrical trapezoid approximation of Type II generalized bell-shaped fuzzy number of is formed.
\end{abstract}

\section{Giriş}

Bulanık kümeler belirsizliğin belirlenmesinde kullanışlıdır. Bununla birlikte bulanık kümelerin gösteriminde kullanılan bulanık fonksiyonlar oldukça detaylı bilgiler sunabilir ve bu durum hesaplama zorluklarına yol açabilir. Çok detaylı bilgiler ve hesaplama zorluklarına neden olan üyelik fonksiyonları yerine

-belirsizliği yeterince içinde barındıran,

- hesaplamalarda daha kolay işlem olanağı taniyan üyelik fonksiyonlarının yakınsamaları bazı uygulamalarda tercih edilebilir [1] .

Bulanık sayının parametrik yakınsamalarında çeşitli yöntemler bulunmaktadır. Bu yöntemlerden bazıları bulanık sayının farklı sol ve sağ fonksiyonlarına sahip, onun en yakın bulanık yakınsamasının merkez ve genişlik değerleri arasındaki farkın azaltılmasına dayanır. Bazl yöntemler ise alan hesabına dayanmaktadır. Örneğin, Ban ve Coroianu, belirsizliği koruyarak ortalama Öklid uzaklığına göre en yakın gerçek aralık, en yakın üçgen (simetrik) bulanık sayı 
ve en yakın (simetrik) yamuk bulanık sayıyı bulmuşlardır [2]. Grzegorzewski ve Winiarska beklenen aralığını koruyan yamuk bulanık sayı yakınsamasını sunmuşlar ve bulanık sayının çekirdeği ve taşıyıcısı için ek gereklilikleri sağlayan yeni operatörler önerilmişlerdir [3]. Zeng ve Li bulanık sayının yakınsamasında ağırlıklı uzaklık kullanarak üçgen yaklaşım sunmuşlardır [4]. Ban vd. belirsizliği ve değeri koruyarak ortalama Öklid uzaklığına göre en yakın yamuk bulanık sayı yakınsamasını oluşturmuşlardır [5]. Chanas bulanık sayının aralık yakınsamasını önermiş, bu önermede bulanık sayı ve aralık arasındaki uzaklık ve bulanık sayı ve yakınsamasının genişlikleri dikkate alınmıştır [6]. Coroianu vd. 0 ile 1 arasında kesin olmayan seviyelere odaklanarak bulanık sayının parçalı doğrusal yaklaşımını sunmuşlardır [7]. Huang vd. differansiyellenebilen yaklaşımlar sunan bir yöntemle bulanık sayının yakınsaması üzerinde çalışmışlardır [8]. Liu ve Lin minimum varyanslı ağırlık fonksiyonu ile bulanık sayının parametreli yakınsamasını oluşturmuşlardır [9]. Coroianua ve Stefanini F-transform kullanarak bulanık sayının yakınsamasını elde etmişlerdir [10]. Nasibov ve Peker bulanık bir sayının parametrik yamuk yakınsamasını sunmuşlardır [11]. Yeh ve Chu Sol-Sağ (LR) tip bulanık sayılar ile yakınsama oluşturmuştur [12]. Wang ve Li basit bulanık sayı üyelik fonksiyonlarını kullanarak bulanı sayı fonksiyonlarının yakınsamalarını sunmuşlardır [13]. Ban vd. ağırlıklandırılmış ortalama Öklit uzaklığı ve Hilbert uzayındaki genel sonuçlara dayanan bir yöntem ile bulanık sayının ağırlıklandırılmış Sol-Sağ (LR) yakınsmasını hesaplamışlardır [14].

Literatürdeki çalışmalarda genel olarak evrensel kümede her bir $x$ 'in bir tane üyelik seviyesine sahip olduğu Tip I bulanık sayısına odaklanılmıştır. Bu çalışmada farklı olarak, her bir $x$ için bulanık sayı şeklinde bir üyelik derecesine olanak tanıyan Tip II bulanık sayısına odaklanılmış ve Tip II genelleştirilmiş çan bulanık sayısının en iyi Tip II parametrik yamuk bulanık sayı yakınsamasının parametreleri elde edilmiştir.

\section{Bulanık Sayı ile İlgili Kavramlar}

$F(E)$ bulanık sayıların uzayı olmak üzere, $A$ bulanık sayisı

$$
A=\bigcup_{\alpha \in[0,1]}\left(\alpha, A_{\alpha}\right)
$$

ile gösterilebilir. Burada $\forall \alpha \in[0,1]: A_{\alpha}=$ $\left[L_{A}(\alpha), R_{A}(\alpha)\right] \subset(-\infty, \infty)$ öyle ki $L:[0,1] \rightarrow(-\infty, \infty)$ monoton azalmayan ve $R:[0,1] \rightarrow(-\infty, \infty)$ monoton artmayan soldan sürekli fonksiyonlardır [11].

Yamuk ve üçgen bulanık sayılar literatürde en çok kullanılan bulanı sayı tiplerindendir. Hesaplamalarda kolaylık sağladığından bulanık sayıların yamuk ve üçgen yakınsamaları tercih edilen yakınsamalar arasındadır.
Şekil 1'de gösterilen yamuk bulanık sayının üyelik fonksiyonu,

$$
\mu_{A}(x)=\left\{\begin{array}{cc}
0, & x \leq a \\
\frac{x-a}{b-a}, & a \leq x \leq b \\
1, & b \leq x \leq c \\
\frac{d-x}{d-c}, & c \leq x \leq d \\
0, & x \geq d
\end{array}\right.
$$

şeklindedir. $x$ 'in bulanık sayısındaki derecesini gösteren $\mu(x),[0,1]$ arasında değerler alır. [a,b,c,d] yamuk bulanık sayısı ele alındığında, [a,b] aralığında monoton artan sol taraf fonksiyonu, [c,d] aralığında monoton azalan sağ taraf fonksiyonu ile tanımlanabilir [7]. $\mathrm{Bu}$ sol ve sağ taraf fonksiyonlarının tersleri de sirasıyla $L_{A}(\alpha)$ ve $R_{A}(\alpha)$ ile gösterilebilir ve alfa seviye aralığının sol ve sağ uç değerlerini verirler:

$$
\begin{aligned}
& L_{A}(\alpha)=a+\alpha(b-a) \\
& R_{A}(\alpha)=d-\alpha(d-c)
\end{aligned}
$$

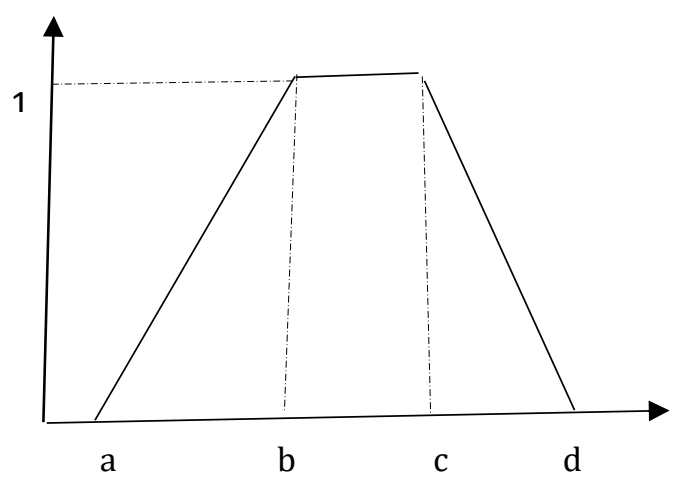

Şekil 1. Yamuk bulanık sayı

Şekil 1'de $L_{A}(1)=R_{A}(1)$ durumunda üçgen bulanık sayı söz konusudur [2]. Bu durum Şekil 2'de gösterilmiştir ve üçgen bulanık sayının üyelik fonksiyonu aşağıdaki gibidir,

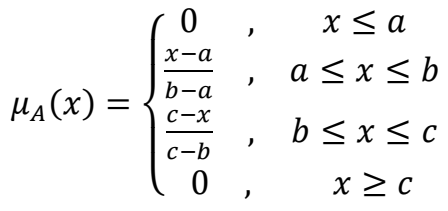

Burada

$$
\begin{aligned}
& L_{A}(\alpha)=a+\alpha(b-a) \\
& R_{A}(\alpha)=c-\alpha(c-b)
\end{aligned}
$$

şeklindedir. 


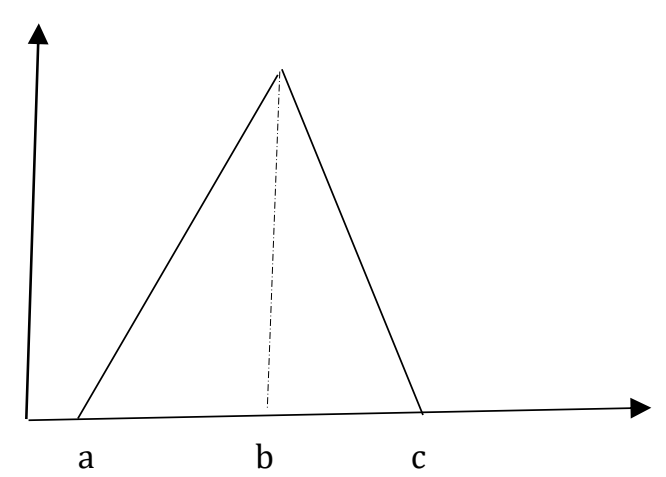

Şekil 2. Üçgen bulanık sayı

Parametrik yamuk bulanık sayının üyelik fonksiyonu

$$
\mu_{A}(x)=\left\{\begin{array}{ccc}
0 & x \leq a \\
1-\left(\frac{b-x}{\sigma}\right)^{s_{L}}, & , & a \leq x \leq b \\
1 & , & b \leq x \leq c \\
1-\left(\frac{x-c}{\beta}\right)^{s_{R}}, & , & c \leq x \leq d \\
0 & , & x \geq d
\end{array}\right.
$$

şeklindedir. Buradaki , $s_{L}>0, s_{R}>0$, bulanık sayının sağ ve sol taraf fonksiyonunun şeklini veren parametrelerdir [11]. Örnek olarak, Şekil 3'de $a=2$, $b=c=10, \sigma=8, \beta=4, s_{L}=1 / 3, s_{R}=2 \quad$ olduğu parametrik üçgen bulanık sayı yer almaktadır.

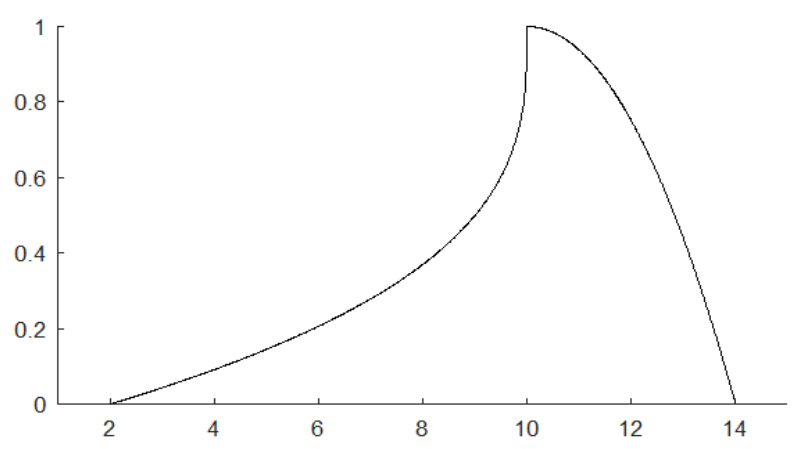

Şekil 3. Parametrik üçgen bulanık sayı

Tip 2 bulanık kümeler, bulanık üyelik fonksiyonu ile karakterize edilebilir; kümenin her elemanı için üyelik değeri [0,1]'de bulanık bir kümedir [13]. Bir başka değişle, Tip I bulanık kümelerinde $x^{\prime}$ in üyelik derecesi tek bir değer iken, Tip II bulanık kümelerinde $x^{\prime}$ in üyelik derecesi Tip I bulanık değerlidir [14]. Şekil 4'te Tip II bulanık sayının iç ve dış fonksiyonları görsel olarak verilmiştir.

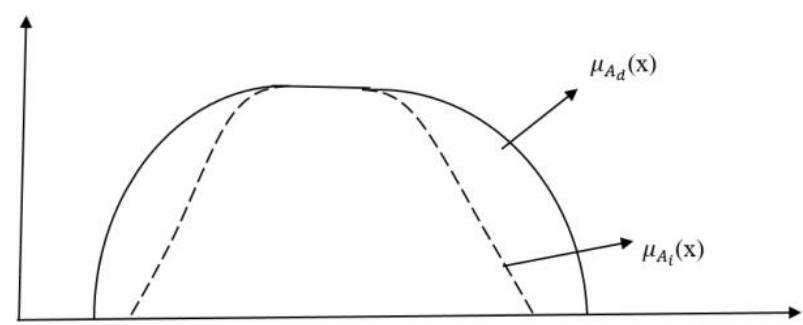

Şekil 4. Tip II bulanık sayı
İç ve dış fonksiyonları genelleştirilmiş çan tipinde olan Tip II bulanık sayısının iç ve dış üyelik fonksiyonları ile sol ve sağ taraf fonksiyonları sirasiyla,

$$
\begin{aligned}
& \mu_{\mathrm{A}_{\mathrm{i}}}(\mathrm{x})= \begin{cases}\frac{1}{1+\left(\frac{c_{1 A_{i}}-\mathrm{x}}{\sigma_{A_{i}}}\right)^{2 P_{L_{i}}}}, & \mathrm{x} \leq c_{1 A_{i}} \\
\frac{1}{1+\left(\frac{x-c_{2 A_{i}}}{\beta_{A_{i}}}\right)^{2 P_{R_{i}}}} & , \quad \mathrm{x} \geq c_{2 A_{i}}\end{cases} \\
& \mu_{\mathrm{A}_{d}}(\mathrm{x})= \begin{cases}\frac{1}{1+\left(\frac{c_{1 A_{d}}-\mathrm{x}}{\sigma_{A_{d}}}\right)^{2 P_{L_{d}}}}, & \mathrm{x} \leq c_{1 A_{d}} \\
\frac{1}{1+\left(\frac{x-c_{2 A_{d}}}{\beta_{A_{d}}}\right)^{2 P_{R_{d}}}} & , \quad \mathrm{x} \geq c_{2 A_{d}}\end{cases} \\
& L_{A_{i}}(\alpha)=c_{1 A_{i}}-\left(\frac{1-\alpha}{\alpha}\right)^{\frac{1}{2 P L_{i}}} \sigma_{A_{i}} \\
& R_{A_{i}}(\alpha)=c_{2 A_{i}}+\left(\frac{1-\alpha}{\alpha}\right)^{\frac{1}{2 P_{R_{i}}}} \beta_{A_{i}} \\
& L_{A_{d}}(\alpha)=c_{1 A_{d}}-\left(\frac{1-\alpha}{\alpha}\right)^{\frac{1}{2 P L_{d}}} \sigma_{A_{d}} \\
& R_{A_{d}}(\alpha)=c_{2 A_{d}}+\left(\frac{1-\alpha}{\alpha}\right)^{\frac{1}{2 P_{R}}} \beta_{A_{d}}
\end{aligned}
$$

şeklindedir [17]. Burada $c_{1 A_{i}}=c_{2 A_{i}}=c_{1 A_{d}}=c_{2 A_{d}}$ 'dir ve makalede aralık Tip II bulanık sayı tanımı dikkate alınmıştır.

\section{Tip II Genelleştirilmiş Çan Şekilli Bulanık Sayısının Tip II Parametik Yamuk Bulanık Sayı Yakınsanması}

$\mathrm{Bu}$ bölümde iç ve dıș fonksiyonları genelleștirilmiș çan olan Tip II $A$ bulanık sayısının, iç ve diş fonksiyonları parametrik yamuk olan Tip II $B$ yakınsaması ele alınmıştır.

Orijinal bulanık sayı ile yakınsaması arasındaki uzaklık hesabında Öklit uzaklığının genişlemesi çalışmalarda kullanılan yöntemlerden birisidir [7]; bu bağlamda aşağıdaki $D$ uzaklığı iki Tip II bulanık sayı arasındaki uzaklık için kullanılmıştır.

$$
D(A, B)=\int_{0}^{1}\left[\begin{array}{c}
\left(L_{A_{\mathrm{I}}}(\alpha)-L_{B_{\mathrm{I}}}(\alpha)\right)^{2} \\
+\left(L_{A_{d}}(\alpha)-L_{B_{d}}(\alpha)\right)^{2} \\
+\left(R_{A_{\mathrm{i}}}(\alpha)-R_{B_{\mathrm{I}}}(\alpha)\right)^{2} \\
+\left(R_{A_{d}}(\alpha)-R_{B_{d}}(\alpha)\right)^{2}
\end{array}\right]^{1 / 2} d \alpha
$$

İç ve dış fonksiyonları parametrik yamuk olan Tip II yakınsamasının ( $B$ sayısı) üyelik fonksiyonları ise 


$$
\begin{aligned}
& \left.\mu_{\mathrm{Bi}_{\mathrm{i}}} \mathrm{x}\right)=\left\{\begin{array}{ccc}
0 & \mathrm{x} \leq c_{1 B_{i}}-\sigma_{B_{i}} \\
1-\left(\frac{c_{1 B_{i}}-\mathrm{x}}{\sigma_{B_{i}}}\right)^{s_{L_{i}}}, & , & c_{1 B_{i}}-\sigma_{B_{i}} \leq \mathrm{x} \leq c_{1 B_{i}} \\
1 & , & c_{1 B_{i}} \leq \mathrm{x} \leq c_{2 B_{i}} \\
1-\left(\frac{x-c_{2 B_{i}}}{\beta_{B_{i}}}\right)^{s_{R_{i}}}, & , & c_{2 B_{i}} \leq \mathrm{x} \leq c_{2 B_{i}}+\beta_{B_{i}} \\
0 & , & \mathrm{x} \geq c_{2 B_{i}}+\beta_{B_{i}}
\end{array}\right. \\
& \mu_{\mathrm{B}_{D}}(\mathrm{x})=\left\{\begin{array}{ccc}
0 & \mathrm{x} \leq c_{1 B_{d}}-\sigma_{B_{d}} \\
1-\left(\frac{c_{1 B_{d}}-x}{\sigma_{B_{d}}}\right)^{s_{L_{d}}}, & , & c_{1 B_{d}}-\sigma_{B_{d}} \leq \mathrm{x} \leq c_{1 B_{d}} \\
1 & , & c_{1 B_{d}} \leq \mathrm{x} \leq c_{2 \mathrm{~d}} \\
1-\left(\frac{\mathrm{x}-c_{2 B_{d}}}{\beta_{B_{d}}}\right)^{S_{R_{d}}}, & , & c_{2 B_{d}} \leq \mathrm{x} \leq c_{2 B_{d}}+\beta_{B_{d}} \\
0 & , & \mathrm{x} \geq c_{2 B_{d}}+\beta_{B_{d}}
\end{array}\right.
\end{aligned}
$$

ile tanımlanmıştır. Buna göre

$$
\begin{gathered}
L_{B_{i}}(\alpha)=c_{1 B_{i}}-(1-\alpha)^{\frac{1}{s_{L_{i}}}} \sigma_{B_{i}} \\
R_{B_{i}}(\alpha)=c_{2 B_{i}}+(1-\alpha)^{\frac{1}{s_{R_{i}}}} \beta_{B_{i}} \\
L_{B_{d}}(\alpha)=c_{1 B_{d}}-(1-\alpha)^{\frac{1}{s_{L_{d}}}} \sigma_{B_{d}} \\
R_{B_{d}}(\alpha)=c_{2 B_{d}}+(1-\alpha)^{\frac{1}{S_{R_{d}}}} \beta_{B_{d}}
\end{gathered}
$$

sağ ve sol taraf fonksiyonları yazılabilir.

Tip 2 bulanık sayısı dikkate alındığında bir $A$ bulanık sayısının verilen $s_{L_{i}}, s_{L_{d}}, s_{R_{i}}, s_{R_{d}}$ değerleri için $B$ yakınsaması (15)'de yer alan uzaklığın karesinin $c_{1 B_{i}}$, $c_{2 B_{i}}, c_{1 B_{d^{\prime}}}, c_{2 B_{d^{\prime}}}, \sigma_{B_{i}}, \beta_{B_{i}}, \sigma_{B_{d}}, \beta_{B_{d}}$ parametrelerine göre en küçük değerinin elde edilmesi ile bulunabilir.

$$
G(A, B)=\int_{0}^{1}\left[\begin{array}{c}
\left(L_{A_{\mathrm{i}}}(\alpha)-L_{B_{\mathrm{i}}}(\alpha)\right)^{2} \\
+\left(L_{A_{d}}(\alpha)-L_{B_{d}}(\alpha)\right)^{2} \\
+\left(R_{A_{\mathrm{i}}}(\alpha)-R_{B_{\mathrm{i}}}(\alpha)\right)^{2} \\
+\left(R_{A_{d}}(\alpha)-R_{B_{d}}(\alpha)\right)^{2}
\end{array}\right] d \alpha
$$

Burada $B$ yakınsaması için uygun $s_{L}, s_{R}$ değerlerinin belirlenmesi için amaç fonksiyonun minimizasyonunda, yakınsamasının çekirdek, sol ve sağ uç değerleri için

$$
\begin{gathered}
c_{1 B_{d}} \leq c_{1 B_{i}} \leq c_{2 B_{i}} \leq c_{2 B_{d}} \\
c_{1 B_{d}}-\sigma_{B_{d}} \leq c_{1 B_{i}}-\sigma_{B_{i}} \leq c_{2 B_{i}}+\beta_{B_{i}} \leq c_{2 B_{d}}+\beta_{B_{d}}
\end{gathered}
$$

eşitsizliklerinin sağlanması beklenir.

Teorem: Verilen $s_{L_{i}}, s_{L_{d^{\prime}}}, s_{R_{i}}, s_{R_{d}}$ değerleri için Tip 2 genelleștirilmiş çan bulanık sayısının (22)'deki $G$ amaç fonksiyonuna göre en yakın Tip II parametrik yamuk yakınsamasının $c_{1 B_{i}}, c_{2 B_{i}}, c_{1 B_{d}}, c_{2 B_{d^{\prime}}}, \sigma_{B_{i}}, \beta_{B_{i}}$, $\sigma_{B_{d}}, \beta_{B_{d}}$ değerleri aşağıdaki gibidir:

$$
\begin{aligned}
& c_{1 B_{i}}=\frac{\sigma_{B_{i}} s_{L_{i}}}{1+s_{L_{i}}}+c_{1 A_{i}}-\sigma_{A_{i}} B\left(\frac{1}{2 P_{L_{i}}}+1,-\frac{1}{2 P_{L_{i}}}+1\right) \\
& c_{2 B_{i}}=-\frac{\beta_{B_{i}} S_{R_{i}}}{1+s_{R_{i}}}+c_{2 A_{i}}+\beta_{A_{i}} B\left(\frac{1}{2 P_{R_{i}}}+1,-\frac{1}{2 P_{R_{i}}}+1\right) \\
& c_{1 B_{d}}=\frac{\sigma_{B d} s_{L_{d}}}{1+s_{L_{d}}}+c_{1 A_{d}}-\sigma_{A_{d}} B\left(\frac{1}{2 P_{L_{d}}}+1,-\frac{1}{2 P_{L_{d}}}+1\right) \\
& c_{2 B_{d}}=-\frac{\beta_{B_{d}} s_{R_{d}}}{1+s_{R_{d}}}+c_{2 A_{d}}+\beta_{A_{d}} B\left(\frac{1}{2 P_{R_{d}}}+1,-\frac{1}{2 P_{R_{d}}}+1\right) \\
& \sigma_{B_{i}}=\left[\begin{array}{c}
\frac{\sigma_{A_{i}}\left(\frac{s_{L_{i}}}{1+s_{L_{i}}} B\left(\frac{1}{2 P_{L_{i}}}+1,-\frac{1}{2 P_{L_{i}}}+1\right)\right)}{-\frac{s_{L_{i}}}{2+s_{L_{i}}}+\left(\frac{s_{L_{i}}}{1+s_{L_{i}}}\right)^{2}} \\
-\frac{\sigma_{A_{i}}\left(B\left(\frac{1}{2 P_{L_{i}}}+\frac{1}{s_{L_{i}}}+1,-\frac{1}{2 P_{L_{i}}}+1\right)\right)}{-\frac{s_{L_{i}}}{2+s_{L_{i}}}+\left(\frac{s_{L_{i}}}{1+s_{L_{i}}}\right)^{2}}
\end{array}\right] \\
& \beta_{B_{i}}=\left[\begin{array}{c}
\frac{\beta_{A_{i}}\left(-\frac{s_{R_{i}}}{1+s_{R_{i}}} B\left(\frac{1}{2 P_{R_{i}}}+1,-\frac{1}{2 P_{R_{i}}}+1\right)\right)}{\frac{s_{R_{i}}}{2+s_{R_{i}}}-\left(\frac{s_{R_{i}}}{1+s_{R_{i}}}\right)^{2}} \\
+\frac{\beta_{A_{i}}\left(B\left(\frac{1}{2 P_{R_{i}}}+\frac{1}{s_{R_{i}}}+1,-\frac{1}{2 P_{R_{i}}}+1\right)\right)}{\frac{s_{R_{i}}}{2+s_{R_{i}}}-\left(\frac{s_{R_{i}}}{1+s_{R_{i}}}\right)^{2}}
\end{array}\right] \\
& \sigma_{B_{d}}=\left[\begin{array}{c}
\frac{\sigma_{A_{d}}\left(\frac{s_{L_{d}}}{1+s_{L_{d}}} B\left(\frac{1}{2 P_{d}}+1,-\frac{1}{2 P_{L_{d}}}+1\right)\right)}{-\frac{s_{L_{d}}}{2+s_{L_{d}}}+\left(\frac{s_{L_{d}}}{1+s_{L_{d}}}\right)^{2}} \\
-\frac{\sigma_{A_{d}}\left(B\left(\frac{1}{2 P_{L_{d}}}+\frac{1}{s_{L_{d}}}+1,-\frac{1}{2 P_{L_{d}}}+1\right)\right)}{-\frac{s_{L_{d}}}{2+s_{L_{d}}}+\left(\frac{s_{L_{d}}}{1+s_{L_{d}}}\right)^{2}}
\end{array}\right] \\
& \beta_{B_{d}}=\left[\begin{array}{c}
\frac{\beta_{A_{d}}\left(-\frac{s_{R_{d}}}{1+s_{R_{d}}} B\left(\frac{1}{2 P_{R_{d}}}+1,-\frac{1}{2 P_{R_{d}}}+1\right)\right)}{\frac{s_{R_{d}}}{2+s_{R_{d}}}-\left(\frac{s_{R_{d}}}{1+s_{R_{d}}}\right)^{2}} \\
+\frac{\beta_{A_{d}}\left(B\left(\frac{1}{2 P_{R_{d}}}+\frac{1}{s_{R_{d}}}+1,-\frac{1}{2 P_{R_{d}}}+1\right)\right)}{\frac{s_{R_{d}}}{2+s_{R_{d}}}-\left(\frac{s_{R_{d}}}{1+s_{R_{d}}}\right)^{2}}
\end{array}\right]
\end{aligned}
$$

Burada $s_{L_{i}}, s_{L_{d}}, s_{R_{i}}, s_{R_{d}}$ değerlerinin seçiminde (23) ve (24) eșitsizliklerini sağlayacak șekilde seçimler yapılmalıdır. Formüllerde $B(*, *)$ beta fonksiyonunu göstermektedir.

İspat: Eşitlik (22)'de yer alan amaç fonksiyonunun minimizasyonu bulabilmek için aşağıdaki eşitliklikler çözülür.

$\frac{\partial G d \alpha}{\partial c_{1 B_{i}}}=0, \frac{\partial G d \alpha}{\partial c_{2 B_{i}}}=0, \frac{\partial G d \alpha}{\partial c_{1 B_{d}}}=0, \frac{\partial G d \alpha}{\partial c_{2 B_{d}}}=0, \frac{\partial G d \alpha}{\partial \sigma_{B_{i}}}=0$,
$\frac{\partial G d \alpha}{\partial \beta_{B_{i}}}=0, \frac{\partial G d \alpha}{\partial \sigma_{B_{d}}}=0, \frac{\partial G d \alpha}{\partial \beta_{B_{d}}}=0$

Bu eşitlikler çözüldüğünde sırasıyla 


$$
\begin{gathered}
2 \int_{0}^{1}\left(c_{1 B_{i}}-(1-\alpha)^{\frac{1}{s_{L_{i}}}} \sigma_{B_{i}}-L_{A_{i}}(\alpha)\right) d \alpha=0 \\
2 \int_{0}^{1}\left(c_{2 B_{i}}+(1-\alpha)^{\frac{1}{s_{R_{i}}}} \beta_{B_{i}}-R_{A_{i}}(\alpha)\right) d \alpha=0 \\
2 \int_{0}^{1}\left(c_{1 B_{d}}-(1-\alpha)^{\frac{1}{s_{L_{d}}}} \sigma_{B_{d}}-L_{A_{d}}(\alpha)\right) d \alpha=0 \\
2 \int_{0}^{1}\left(c_{2 B_{d}}+(1-\alpha)^{\frac{1}{S_{R_{d}}}} \beta_{B_{d}}-R_{A_{d}}(\alpha)\right) d \alpha=0 \\
-2 \int_{0}^{1}\left(c_{1 B_{i}}-(1-\alpha)^{\frac{1}{s_{L_{i}}}} \sigma_{B_{i}}-L_{A_{i}}(\alpha)\right)(1-\alpha)^{\frac{1}{s_{L_{i}}}} d \alpha=0 \\
2 \int_{0}^{1}\left(c_{2 B_{i}}+(1-\alpha)^{\frac{1}{s_{i}}} \beta_{B_{i}}-R_{A_{i}}(\alpha)\right)(1-\alpha)^{\frac{1}{s_{R_{i}}}} d \alpha=0 \\
-2 \int_{0}^{1}\left(c_{1 B_{d}}-(1-\alpha)^{\frac{1}{s_{L_{d}}}} \sigma_{B_{d}}-L_{A_{d}}(\alpha)\right)(1-\alpha)^{\frac{1}{s_{L_{d}}}} d \alpha=0 \\
2 \int_{0}^{1}\left(c_{2 B_{d}}+(1-\alpha)^{\frac{1}{s_{R_{d}}}} \beta_{B_{d}}-R_{A_{d}}(\alpha)\right)(1-\alpha)^{\frac{1}{s_{R_{d}}}} d \alpha=0
\end{gathered}
$$

elde edilir.

Genelleştirilmiş çan Tip II bulanık sayısı için

$$
2 \int_{0}^{1}\left(c_{1 B_{i}}-(1-\alpha)^{\frac{1}{s_{L_{i}}}} \sigma_{B_{i}}-L_{A_{i}}(\alpha)\right) d \alpha=0
$$

eşitliği çözüldüğünde

$c_{1 B_{i}}+\sigma_{B_{i}} \frac{(1-\alpha)^{1+\frac{1}{s_{L_{i}}}}}{1+\frac{1}{s_{L_{i}}}} \mid-\int_{0}^{1}\left(c_{1 A_{i}}-\left(\frac{1-\alpha}{\alpha}\right)^{\frac{1}{2 P L_{i}}} \sigma_{A_{i}}\right) d \alpha=$ 0

ve buradan

$$
c_{1 B_{i}}=\frac{\sigma_{B_{i}} S_{L_{i}}}{1+s_{L_{i}}}+c_{1 A_{i}}-\sigma_{A_{i}} B\left(\frac{1}{2 P_{L_{i}}}+1,-\frac{1}{2 P_{L_{i}}}+1\right)
$$

elde edilir.

İç fonksiyonun sol yayılması için

$$
\begin{aligned}
& -2 \int_{0}^{1}\left(c_{1 B_{i}}-(1-\alpha)^{\frac{1}{S_{L_{i}}}} \sigma_{B_{i}}-L_{A_{i}}(\alpha)\right)(1-\alpha)^{\frac{1}{S_{L_{i}}}} d \alpha \\
& =0
\end{aligned}
$$

eşitliği çözüldüğünde

$$
-\frac{(1-\alpha)^{1+\frac{1}{s_{L_{i}}}}}{1+\frac{1}{s_{L_{i}}}}\left|+\sigma_{B_{i}} \frac{(1-\alpha)^{1+\frac{2}{s_{L_{i}}}}}{1+\frac{2}{s_{L_{i}}}}\right|
$$

$$
-\int_{0}^{1}\left(c_{1 A_{i}}-\left(\frac{1-\alpha}{\alpha}\right)^{\frac{1}{2 P_{L_{i}}}} \sigma_{A_{i}}\right)(1-\alpha)^{\frac{1}{S_{L_{i}}}} d \alpha=0
$$

bulunur ve bu eşitlikte $c_{1 B_{i}}$ yerine

$$
\frac{\sigma_{B_{i}} S_{L_{i}}}{1+S_{L_{i}}}+c_{1 A_{i}}-\sigma_{A_{i}} B\left(\frac{1}{2 P_{L_{i}}}+1,-\frac{1}{2 P_{L_{i}}}+1\right)
$$

konulduğunda

$$
\begin{aligned}
\frac{s_{L_{i}}}{1+s_{L_{i}}}\left(\frac{\sigma_{B_{i}} S_{L_{i}}}{1+s_{L_{i}}}+\right. & \left.c_{1 A_{i}}-\sigma_{A_{i}} B\left(\frac{1}{2 P_{L_{i}}}+1,-\frac{1}{2 P_{L_{i}}}+1\right)\right) \\
& -\frac{\sigma_{B_{i}} s_{L_{i}}}{2+s_{L_{i}}}-c_{1 A_{i}} \frac{s_{L_{i}}}{1+s_{L_{i}}} \\
& +\sigma_{A_{i}} B\left(\frac{1}{2 P_{L_{i}}}+\frac{1}{s_{L_{i}}}+1,-\frac{1}{2 P_{L_{i}}}+1\right) \\
& =0
\end{aligned}
$$

elde edilir ve buradan

$$
\sigma_{B_{i}}=\left[\begin{array}{c}
\frac{\sigma_{A_{i}}\left(\frac{s_{L_{i}}}{1+s_{L_{i}}} B\left(\frac{1}{2 P_{L_{i}}}+1,-\frac{1}{2 P_{L_{i}}}+1\right)\right)}{-\frac{s_{L_{i}}}{2+s_{L_{i}}}+\left(\frac{s_{L_{i}}}{1+s_{L_{i}}}\right)^{2}} \\
-\frac{\sigma_{A_{i}} B\left(\frac{1}{2 P_{L_{i}}}+\frac{1}{s_{L_{i}}}+1,-\frac{1}{2 P_{L_{i}}}+1\right)}{-\frac{s_{L_{i}}}{2+s_{L_{i}}}+\left(\frac{s_{L_{i}}}{1+s_{L_{i}}}\right)^{2}}
\end{array}\right]
$$

elde edilir.

Sağ iç fonksiyon dikkate alındığında ise

$$
\begin{gathered}
2 \int_{0}^{1}\left(c_{2 B_{i}}+(1-\alpha)^{\frac{1}{S_{R_{i}}}} \beta_{B_{i}}-R_{A_{i}}(\alpha)\right) d \alpha=0 \\
c_{2 B_{i}}-\beta_{B_{i}} \frac{(1-\alpha)^{1+\frac{1}{S_{L_{i}}}}}{1+\frac{1}{s_{L_{i}}}} \mid \\
-\int_{0}^{1}\left(c_{2 A_{i}}+\left(\frac{1-\alpha}{\alpha}\right)^{\frac{1}{2 P_{L_{i}}}} \beta_{A_{i}}\right) d \alpha=0
\end{gathered}
$$

eşitliği ve buradan

$$
c_{2 B_{i}}=-\frac{\beta_{B_{i}} S_{R_{i}}}{1+s_{R_{i}}}+c_{2 A_{i}}+\beta_{A_{i}} B\left(\frac{1}{2 P_{R_{i}}}+1,-\frac{1}{2 P_{R_{i}}}+1\right)
$$

elde edilir.

Sağ iç yayllma içinse

$$
\begin{aligned}
& 2 \int_{0}^{1}\left(c_{2 B_{i}}+(1-\alpha)^{\frac{1}{S_{R_{i}}}} \beta_{B_{i}}-R_{A_{i}}(\alpha)\right)(1-\alpha)^{\frac{1}{S_{R_{i}}}} d \alpha \\
= & 0
\end{aligned}
$$

eşitliği dikkate alınır ve 
$-c_{2 B_{i}} \frac{(1-\alpha)^{1+\frac{1}{s_{R_{i}}}}}{1+\frac{1}{s_{R_{i}}}} \mid-\beta_{B_{i}} \frac{(1-\alpha)^{1+\frac{2}{s_{R_{i}}}}}{1+\frac{2}{s_{R_{i}}}}$

$+c_{2 A_{i}} \frac{(1-\alpha)^{1+\frac{1}{s_{R_{i}}}}}{1+\frac{1}{s_{R_{i}}}}$

$-\beta_{A_{i}} B\left(\frac{1}{2 P_{R_{i}}}+\frac{1}{S_{R_{i}}}+1,-\frac{1}{2 P_{R_{i}}}+\frac{1}{S_{R_{i}}}+1\right)=0$

elde edilerek $c_{2 B_{i}}$ bu eşitlikte yerine konulur ve aşağıdaki eşitsizliğe ulaşılır,

$$
\begin{aligned}
& \frac{s_{R_{i}}}{1+s_{R_{i}}}\left(-\frac{\beta_{B_{i}} s_{R_{i}}}{1+s_{R_{i}}}+c_{2 A_{i}}+\beta_{A_{i}} B\left(\frac{1}{2 P_{R_{i}}}+1,-\frac{1}{2 P_{R_{i}}}+1\right)\right) \\
+ & \beta_{B_{i}} \frac{s_{R_{i}}}{2+s_{R_{i}}}-c_{2 A_{i}} \frac{s_{R_{i}}}{1+s_{R_{i}}} \\
- & \beta_{A_{i}} B\left(\frac{1}{2 P_{R_{i}}}+\frac{1}{s_{R_{i}}}+1,-\frac{1}{2 P_{R_{i}}}+\frac{1}{s_{R_{i}}}+1\right)=0
\end{aligned}
$$

Buradan

$$
\begin{gathered}
\beta_{B_{i}}=\frac{\beta_{A_{i}}\left(-\frac{s_{R_{i}}}{1+s_{R_{i}}} B\left(\frac{1}{2 P_{R_{i}}}+1,-\frac{1}{2 P_{R_{i}}}+1\right)\right)}{\frac{\frac{s_{R_{i}}}{2+s_{R_{i}}}-\left(\frac{s_{R_{i}}}{1+s_{R_{i}}}\right)^{2}}{+\frac{\beta_{A_{i}} B\left(\frac{1}{2 P_{R_{i}}}+\frac{1}{s_{R_{i}}}+1,-\frac{1}{2 P_{R_{i}}}+1\right)}{\frac{s_{R_{i}}}{2+s_{R_{i}}}-\left(\frac{s_{R_{i}}}{1+s_{R_{i}}}\right)^{2}}}}
\end{gathered}
$$

eşitliği sağlanır.

Benzer şekilde $c_{1 B_{d}}, c_{2 B_{d}}, \sigma_{B_{d}}, \beta_{B_{d}}$ için eşitlikler elde edilir.

Örnek: İç ve dış fonksiyonları genelleștirilmiş çan şeklinde olan aşağıdaki Tip II bulanık sayısı verilsin.

$$
\begin{aligned}
& \mu_{\mathrm{A}_{\mathrm{i}}}(\mathrm{x})= \begin{cases}\frac{1}{1+\left(\frac{8-\mathrm{x}}{3}\right)^{4}}, & \mathrm{x} \leq 8 \\
\frac{1}{1+\left(\frac{x-8}{2}\right)^{6}}, & \mathrm{x} \geq 8\end{cases} \\
& \mu_{\mathrm{A}_{d}}(\mathrm{x})= \begin{cases}\frac{1}{1+\left(\frac{8-\mathrm{x}}{6}\right)^{4}}, & \mathrm{x} \leq 8 \\
\frac{1}{1+\left(\frac{x-8}{4}\right)^{6}}, & \mathrm{x} \geq 8\end{cases}
\end{aligned}
$$

Buna göre Tip II bulanık sayısının sol ve sağ taraf fonksiyonları

$$
\begin{aligned}
& L_{A_{i}}(\alpha)=8-\left(\frac{1-\alpha}{\alpha}\right)^{\frac{1}{4}} 3 \\
& R_{A_{i}}(\alpha)=8+\left(\frac{1-\alpha}{\alpha}\right)^{\frac{1}{6}} 2 \\
& L_{A_{d}}(\alpha)=8-\left(\frac{1-\alpha}{\alpha}\right)^{\frac{1}{4}} 6
\end{aligned}
$$

$$
R_{A_{d}}(\alpha)=8+\left(\frac{1-\alpha}{\alpha}\right)^{\frac{1}{6}} 4
$$

şeklindedir.

Tip II $A$ bulanık sayısının Tip II parametrik yamuk yakınsamasının parametreleri

$$
\begin{aligned}
c_{1 B_{i}}= & \frac{4.944996(0.8)}{1+0.8}+8-3 B\left(\frac{5}{4}, \frac{3}{4}\right)=6.865676 \\
c_{2 B_{i}}= & -\frac{2.551156(2.2)}{1+2.2}+8+2 B\left(\frac{7}{6}, \frac{5}{6}\right)=8.34048 \\
c_{1 B_{d}}= & \frac{9.889992(0.8)}{1+0.8}+8-6 B\left(\frac{5}{4}, \frac{3}{4}\right)=5.731352 \\
c_{2 B_{d}}= & -\frac{5.102313(2.2)}{1+2.2}+8+4 B\left(\frac{7}{6}, \frac{5}{6}\right)=8.68096 \\
\sigma_{B_{i}}= & \frac{3\left(\frac{0.8}{1+0.8} B\left(\frac{5}{4}, \frac{3}{4}\right)-B\left(\frac{10}{4}, \frac{3}{4}\right)\right)}{-\frac{0.8}{2.8}+\left(\frac{0.8}{1.8}\right)^{2}}=4.944996 \\
\beta_{B_{i}}= & \frac{2\left(-\frac{2.2}{1+2.2} B\left(\frac{7}{6}, \frac{5}{6}\right)+B\left(\frac{1}{6}+\frac{1}{2.2}+1, \frac{5}{6}\right)\right)}{\frac{2.2}{4.2}-\left(\frac{2.2}{3.2}\right)^{2}} \\
= & 2.551156 \\
\sigma_{B_{d}}= & \frac{6\left(\frac{0.8}{1+0.8} B\left(\frac{5}{4}, \frac{3}{4}\right)-B\left(\frac{10}{4}, \frac{3}{4}\right)\right)}{-\frac{0,8}{2.8}+\left(\frac{0.8}{1.8}\right)^{2}}=9.889992 \\
\beta_{B_{d}}= & \frac{4\left(-\frac{2.2}{1+2.2} B\left(\frac{7}{6}, \frac{5}{6}\right)+B\left(\frac{1}{6}+\frac{1}{2.2}+1, \frac{5}{6}\right)\right)}{\frac{2.2}{4.2}-\left(\frac{2.2}{3.2}\right)^{2}} \\
= & 5.102313
\end{aligned}
$$

Bu parametreler kullanarak Tip II parametrik yamuk bulanık sayısının $L_{A_{i}}(\alpha), L_{A_{d}}(\alpha), R_{A_{i}}(\alpha), R_{A_{d}}(\alpha)$ fonksiyonları

$$
\begin{aligned}
& L_{A_{i}}(\alpha)=6.865676-4.944996(1-\alpha)^{\frac{1}{0.8}} \\
& R_{A_{i}}(\alpha)=8.34048+2.551156(1-\alpha)^{\frac{1}{2.2}} \\
& L_{A_{d}}(\alpha)=5.731352-9.889992(1-\alpha)^{\frac{1}{0.8}} \\
& R_{A_{d}}(\alpha)=8.68096+5.102313(1-\alpha)^{\frac{1}{2.2}}
\end{aligned}
$$

şeklindedir. 


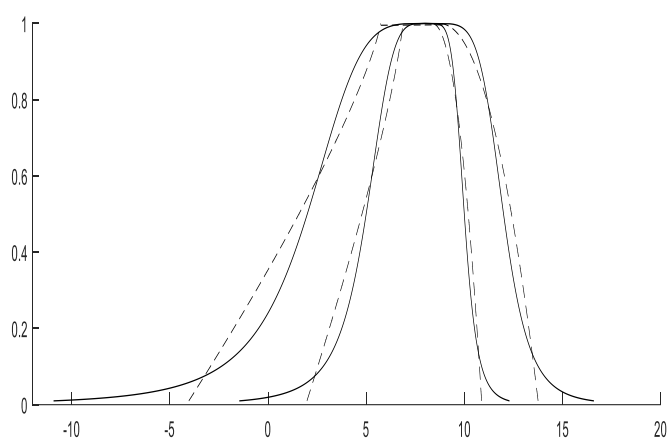

Şekil 5: Tip II genelleştirilmiş çan şekilli bulanık sayısının Tip II parametrik yamuk bulanık sayı yakınsaması

Örnekte verilen Tip II genelleştirilmiş çan bulanık sayısı ile Tip II parametrik yamuk bulanık sayı yakınsaması Şekil 5'te verilmiştir.

\section{Tartışma ve Sonuç}

Tip II bulanık sayısı $x$ değerinin üyelik derecesinin tek bir sayı olması yerine iç ve dış üyelik fonksiyonları yardımıyla belli bir aralıkta üyelik derecelerine sahip olmaya olanak tanır. Bu çalışmada tip II bulanık sayılarına odaklanarak iç ve dış fonksiyonları genelleştirilmiş çan şekilli fonksiyonlardan oluşan tip II bulanık sayısının üyelik fonksiyonları parametrik yamuk olan Tip II bulanık sayısının yakınsaması yapılmıştır. Daha karmaşık yapıdaki bulanık sayının daha basit hale getirilmesi ve çalışmalarda işlem kolaylığı sağlanması amaçlanmıştır. Yakınsamanın oluşturulması aşamasında parametrik bulanık sayının sağ ve sol şeklini veren değerlerin seçimi merkez ve uç değerlerin sağ merkez ve uç değerlerinden daha küçük olması koşulunu koruyacak şekilde seçimlerin yapılmasına olanak sağlayacak merkez ve yayılma formülleri elde edilmiștir.

\section{Kaynakça}

[1] Grzegorzewski, P. 2013. Fuzzy number approximation via shadowed sets. Information Sciences, 25(2013), 35-46.

[2] Ban, A.I., Coroianu, L. 2012. Nearest interval, triangular and trapezoidal approximation of a fuzzy number preserving ambiguity, International Journal of Approximate Reasoning, 53(2012), 805-836.

[3] Grzegorzewski, P., Winiarska, K.P. 2014. Natural trapezoidal approximations of fuzzy numbers. Fuzzy Sets and Systems, 250(2014), 90-109.

[4] Zeng, W., Li, H. 2007. Weighted triangular approximation of fuzzy numbers. International
Journal of Approximate Reasoning, 46(2007), 137-150.

[5] Ban, A., Brândaş, A., Coroianu, L., Negruţiu, C., Nica 0. 2011. Approximations of fuzzy numbers by trapezoidal fuzzy numbers preserving the ambiguity and value. Computers and Mathematics with Applications, 61 (2011) 1379-1401.

[6] Chanas, S. 2001. On the interval approximation of a fuzzy number. Fuzzy Sets and Systems, 122 (2001) 353-356.

[7] Coroianu, L., Gagolewski, M., Grzegorzewski, P. 2013. Nearest piecewise linear approximation of fuzzy numbers. Fuzzy Sets and Systems, 233(2013), 26-51.

[8] Huang, H., Wu, C., Xie, J., Zhang, D. 2017. Approximation of fuzzy numbers using the convolution method. Fuzzy Sets and Systems, 310(2017), 14-46.

[9] Liu, X., Lin, H. 2007. Parameterized approximation of fuzzy number with minimum variance weighting functions. Mathematical and Computer Modelling, 46(2007) 1398-1409.

[10] Coroianu, L., Stefanini, L. 2016. General approximation of fuzzy numbers by F-transform. Fuzzy Sets and Systems, 288(2016) 46-74.

[11] Nasibov, E.N., Peker, S. 2008. On the nearest parametric approximation of a fuzzy number. Fuzzy Sets and Systems, 159 (2008) 1365-1375.

[12] Yeh, C.T., Chu, H.M. 2014. Approximations by LRtype fuzzy numbers. Fuzzy Sets and Systems, 257(2014), 23-40.

[13] Wang, G., Li. 2017. Approximations of Fuzzy Number by Step Type Fuzzy Number, 310 (2017), 47-59.

[14] Ban. A.I., Coroianu, L., Khastan. A. 2016. Conditioned Weighted L-R approximations of fuzzy numbers. Fuzzy Sets and systems, 283 (2016), 56-82.

[15] Karnik, N.N., Mendel, J.M. 2000. Operations on type-2 fuzzy sets. Fuzzy Sets and Systems, 122 (2000), 327-348.

[16] Ngan, S.C. 2018. Revisiting fuzzy set operations: A rational approach for designing set operators for type-2 fuzzy sets and type-2 like fuzzy sets. Expert Systems with Applications, 107 (2018) 255-284.

[17] Feng G. 2010. Analysis and Synthesis of Fuzzy Control Systems: A Model Based Approach. CRC Press, $281 \mathrm{~s}$. 